\title{
Effects of sevoflurane exposure on oligodendrocyte differentiation and myelination in white matter of preterm-equivalent rats
}

\section{Ziyi Wu}

Shengjing Hospital of China Medical University

Qiushi Gao

Shengjing Hospital of China Medical University

Xingyue Li

Shengjing Hospital of China Medical University

Hang Xue

Shengjing Hospital of China Medical University

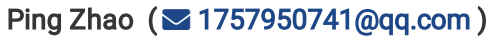

Shengjing Hospital of China Medical University

\section{Research}

Keywords: sevoflurane, preterm neonates, white matter, oligodendrocyte maturation, myelination

Posted Date: November 25th, 2019

DOI: https://doi.org/10.21203/rs.2.17666/v1

License: @ (i) This work is licensed under a Creative Commons Attribution 4.0 International License. Read Full License 


\section{Abstract}

Background: General anesthetics may impose significant neurocognitive risks on the immature brain. Since preterm infants often need anesthesia for surgical or diagnostic procedures, exploring the appropriate anesthetic dose is necessary for procedural needs and for the safety of brain development. As brain injury in preterm neonates has a particular predilection for cerebral white matter, we studied the effects of different concentrations of sevoflurane on oligodendrocyte maturation and myelination in a preterm-equivalent rat model.

Methods: Rats at postnatal day 2 were exposed to 3.3\% (approximately 1 minimum alveolar concentration [MAC]) or $4.9 \%$ (approximately 1.5 MAC) sevoflurane for 2 hours. Changes in oligodendrocyte differentiation and myelin integrity in white matter were evaluated by immunofluorescence with stagespecific markers. Neurobehavioral function was examined by open field test and Morris water maze tests. As prominent hallmarks of white matter injury, microgliosis and astrogliosis were investigated in specific white matter regions.

Results: In the $4.9 \%$ sevoflurane-treatment group, decreased differentiation of oligodendrocytes indicated arrested oligodendrocyte maturation, resulting in hypomyelination and axonal damage in the developing white matter of the rat brain. Treatment increased anxiety-like behaviour and caused learning and memory impairments in adolescent rats. Microglia activation and astrogliosis were also observed at $12 \mathrm{~h}$ post anesthesia. No differences were seen between the control and $3.3 \%$ sevoflurane groups.

Conclusions: Sevoflurane exposure in preterm-equivalent rats dose-dependently disrupted oligodendrocyte maturation and hypomyelination and impaired function. Microgliosis and astrogliosis might contribute to white matter damage.

\section{Background}

The detrimental effects of anesthetic agents on the developing brain have been noted. Most studies of anesthesia-induced developmental neurotoxicity have been conducted in foetal and full-term neonatal animal studies[1]. The World Health Organization estimates that about 15 million babies, more than 1 in 10 live births, are born preterm (delivery before 37 weeks of gestation) worldwide each year[2]. Preterm babies might require diagnostic tests and surgical procedures that use administration of anesthesia[3]. However, whether preterm infants receiving anesthesia are at high risk of adverse neurodevelopmental outcomes as infants born at term is unknown.

White matter occupies roughly half the brain volume and acts in relaying and coordinating communication between different brain regions. White matter is increasingly recognized as critical for cognition[4]. A clinical study showed that children who had anesthesia and surgery during infancy displayed broadly distributed, decreased white matter integrity and volume[5]. White matter in preterm infants is particularly vulnerable to injury due to crucial development processes taking place during the third trimester of pregnancy in humans[6]. Currently, the most common form of brain injury in prematurely born infants is diffuse white matter damage (WMD).

White matter is mainly composed of oligodendrocytes (OLs) and myelinated axons. In the central nervous system (CNS), mature OLs extend processes to touch and wrap neuronal axons, forming a myelin sheath to facilitate electrical propagation and preserve axonal integrity. Preclinical and epidemiological studies demonstrate a clear association between exposure to hypoxic or inflammatory insults at critical points in development and preterm brain injury. Such perinatal insults negatively impact the oligodendrocyte maturation and myelination process, causing long-term neurobehavioral changes. N-methyl-Daspartate and g-aminobutyric acid (GABA) receptors in the oligodendrocyte lineage cells receive excitatory and inhibitory synaptic input signals mediated by glutamate and GABA[7, 8]. Endogenous GABA greatly reduces the number of oligodendrocyte lineage cells, correlating with a reduction in the amount of myelination in vitro[8]. Sevoflurane, the most widely used inhaled anesthetic for full-term and premature neonates, acts as g-aminobutyric acid type $A$ (GABA A $_{\text {) }}$ receptor agonist[9]. Thus, we hypothesized that sevoflurane would have negative impacts on oligodendrocyte maturation and myelination.

To eliminate influences of various confounding factors of human studies, we use a rigorously controlled preterm-equivalent animal model. In postpartum day 2 (P2) rat pups, the stage of brain development coincides with the high-risk period of white matter injury (23-32 weeks of gestation) for human preterm neonates[10]. Use of $\mathrm{P} 1-3$ rodents to model the preterm infant brain is supported by comparative studies of brain growth and oligodendrocyte maturation[11-13]. In this study, we investigated the effects of a moderate duration ( $2 \mathrm{~h}$ ) of sevoflurane at low (3.3\%) and high concentrations (4.9\%) in P2 rats on OL maturation, myelination, and long-term neurobehavioral outcomes. Given the key roles of activated microglia and astroglia in regulation of oligodendrocyte lineage cells, myelination, remyelination, microgliosis, and astrogliosis were also determined.

\section{Methods}

\section{Animals and experimental design}

Experimental animal work used newborn Sprague-Dawley rats bred at the Laboratory Animal Care Committee of China Medical University, Shenyang, China (2016PS028K) and was in accordance with the Guidelines for the Care and Use of Laboratory Animals from the National Institutes of Health (USA). Dams and pups were housed in standard conditions with a 12-h light/dark cycle at $23 \pm 1{ }^{\circ} \mathrm{C}$ and ad libitum access to food and water. Only litters with $10-14$ pups were selected for experiments to avoid effects due to extreme variations in litter size. All procedures were made to minimize animal pain or discomfort.

P2 rats were randomly divided into three groups ( $n=27$ per group): control $(\mathrm{CON})$, low concentration sevoflurane (3.3\% SEV), and high concentration sevoflurane (4.9\% SEV). Rat pups were placed in an anesthesia container with concentrations of sevoflurane, oxygen, and carbon dioxide monitored using a gas monitor (Datex-Ohmeda Inc., Tewksbury, MA, USA). Rat pups in 3.3\% SEV and $4.9 \%$ SEV were respectively administered $3.3 \%$ and $4.9 \%$ sevoflurane in a humidified $30 \%$ oxygen carrier gas with total gas flow $2 \mathrm{~L} \mathrm{~min}^{-1}$ for $2 \mathrm{~h}$. The CON group received humidified $30 \%$ oxygen for $2 \mathrm{~h}$. We chose $3.3 \%$ because it 
roughly represents $1 \mathrm{MAC}$ value in 2-day-old rats[14], and 4.9\% is equal to $1.5 \mathrm{MAC}$. Arterial blood gases and blood glucose were measured at the end of the 2$h$ aneasthetic exposure ( $n=5$ for each group.).

At $12 \mathrm{~h}$ post anesthesia, P14, and P28, five rats from each group were sacrificed and the brains were removed. From P29, twelve rats from each group were used for behavioural tests. Experimental schedules are schematically represented in Fig.1.

\section{Tissue preparation}

Under deep pentobarbital anesthesia, animals were perfused transcardially with phosphate-buffered saline (PBS) followed by $4 \%$ paraformaldehyde. Brains were left overnight at $4^{\circ} \mathrm{C}$ and embedded in paraffin after dehydration in graded ethanol. For each animal and staining procedure, at least three equivalent sections covering the striatum were analysed.

\section{Immunofluorescence}

After dewaxing and heating in citrate buffer, sections were incubated $30 \mathrm{~min}$ in blocking buffer (PBS containing $10 \%$ bovine serum albumin). A $4^{\circ} \mathrm{C}$ overnight incubation was performed with one or two of the following antibodies: anti-CNPase (Abcam, 1:100), anti-MBP (Cell Signalling, 1:50), anti-SMI32 (BioLegend, 1:50), anti-lba1 (Proteintech, 1:50), or anti-GFAP (Abcam, 1:100). Sections were rinsed and incubated with secondary antibodies for 3-4 $\mathrm{h}$ at room temperature, followed by DAPI counterstaining after three washes.

\section{Open field test}

Open field test (OFT) was used to evaluate locomotor activity and anxiety-like behaviour in a novel environment on day P29. The open field apparatus was a square, opaque acrylic container $(100 \times 100 \times 50 \mathrm{~cm})$ and a video camera was placed above the arena to track movement. Individual rat was allowed to explore the open field for a period of $10 \mathrm{~min}$ for each trial. Between testing trials, testing apparatuses were thoroughly cleaned. The exploratory behaviour of animals (including velocity, time in central $50 \times 50 \mathrm{~cm}$ area, and zone transitions) was recorded and analysed by EthoVision XT software (Noldus, Wageningen, The Netherlands).

\section{Morris water maze tests}

Morris water maze (MWM) tests were used to evaluate the ability of spatial learning and memory on days P31-P36. Briefly, a round pool (diameter, $160 \mathrm{~cm}$; depth, $60 \mathrm{~cm})$ was filled with warm $\left(20 \pm 1^{\circ} \mathrm{C}\right)$ water to a height of $1.5 \mathrm{~cm}$ above the surface of a 12-cm-diameter platform in the second quadrant. The tests consisted of two phases: the place trial with four trials per day for 5 days and the probe trial on day 6 . Each rat was successively released into the water from one of the quadrants to search the hidden platform. If a rat succeeded to find the platform within $90 \mathrm{~s}$, it was forced to remain on the platform for $20 \mathrm{~s}$ and the time taken to find the platform was defined as the escape latency. If a rat did not find the platform within $90 \mathrm{~s}$, it was guided to the platform and was forced to stay there for $20 \mathrm{~s}$. in the probe trial, the submerged platform was removed and the rat was released from the opposite quadrant and allowed to swim for $90 \mathrm{~s}$ The number of original platform crossings in the second quadrant was recorded. A video surveillance system (Shanghai Mobile Datum Ltd, Shanghai City, China) recorded the swimming motions of animals.

\section{Statistical analysis}

All data were analysed using GraphPad Prism 7 (GraphPad Prism Software, Inc., CA, USA). All continuous variables were tested for assumption of normality Shapiro-Wilk's test and equal variances by Bartlett's test. If assumption of normality and equal variances were met, one-way analysis of variance (ANOVA) followed by Dunnett's multiple comparisons test was used for data analysis. Otherwise, Kruskal-Wallis test followed by Dunn's multiple comparisons test was used. Results of escape latency were analysed using two-way ANOVA for repeated measurements. $P<0.05$ was considered statistically significant.

\section{Results}

\section{Physiologic parameters}

As shown in Table 1, arterial blood gases values and blood glucose levels were within the normal physiologic range. There were no significant differences between the low and high concentrations of sevoflurane treatment groups on any measured variables for arterial blood gases values and blood glucose levels. Taking these measures reduces the possibility that sevoflurane-induced neurotoxicity in the immature brains was caused by physiologic side effects (e.g. hypoglycemia, hypoxia and hypercapnia).

\section{Effects of sevoflurane exposure on rat brain and body growth patterns}

Sevoflurane had no effect on brain or body weight or brain-to-body weight ratio at P2, P14, and P28. See Table 2 for details.

\section{High-concentration sevoflurane exposure impaired oligodendrocyte differentiation/maturation in white matter}

To assess oligodendrocyte differentiation in rats exposed to sevoflurane, we performed immunofluorescence of CNPase, marker of early myelinating OL. Compared with the CON group, rats in the $4.9 \%$ SEV group had decreased CNPase immunoreactivity in the cingulum (Fig. 2; one-way ANOVA followed by Dunnett's multiple comparisons test, $P<0.01$ ), external capsule (Fig. 2; one-way ANOVA followed by Dunnett's multiple comparisons test, $P<0.05$ ), and striatum (Fig. 2; one-way ANOVA followed by Dunnett's multiple comparisons test, $P<0.05$ ), indicating impaired oligodendrocyte differentiation/maturation. No differences were seen between the CON and 3.3\% SEV groups in CNPase immunoreactivity. 
High-concentration sevoflurane exposure induced myelin loss and axonal damage in white matter

White matter integrity was evaluated by immunofluorescence double labelling with MBP as a marker of myelin integrity, and the abnormal nonphosphorylated neurofilament protein, SMI32 as a marker of axonal damage. WMD was expressed as mean ratio of SMI32 to MBP immunostaining. In the CON group, SMI32 immunoreactivity and high MBP expression was rare (Fig. 3). The 4.9\% SEV group showed increased SMI32 expression and a loss of MBP intensity in the corpus callosum (Fig. 3a, e; one-way ANOVA followed by Dunnett's multiple comparisons test, $P<0.001$ ), cingulum (Fig. 3b, e; Kruskal-Wallis test followed by Dunn's multiple comparisons test, $P=0.002$ ), external capsule (Fig. 3c, e; Kruskal-Wallis test followed by Dunn's multiple comparisons test, $P=0.002$ ), and striatum (Fig. 3d, e; Kruskal-Wallis test followed by Dunn's multiple comparisons test, $P<0.001$ ). The increased fluorescence intensity ratio of SMI32/MBP indicated myelin loss accompanied by axonal damage in specific white matter regions. No differences were seen between the CON and $3.3 \%$ SEV groups in expression of SMI32 or MBP (Fig. 3).

\section{High-concentration sevoflurane altered behaviour and cognitive performance in rats}

OFTs showed no differences among groups in velocity (Fig. 4a; one-way ANOVA followed by Dunnett's multiple comparisons test). In comparison with control rats, rats treated with high sevoflurane used significantly less time in the centre (Fig. 4b; Kruskal-Wallis test followed by Dunn's multiple comparisons test, $P=$ 0.013) and zone transitions (Fig. 4c; Kruskal-Wallis test followed by Dunn's multiple comparisons test, $P=0.023$ ), which are signs of anxiety-like behaviour. Spatial learning and memory ability of rats were assessed by MWM tests. No significant differences were seen among groups in swimming speed (Fig. 4d; two-way ANOVA for repeated measurements followed by Dunnett's multiple comparisons test), indicating that sevoflurane did not cause motor dysfunction in rats. Impairment in learning abilities was observed in the $4.9 \%$ SEV group as indicated by a longer escape latency than the CON group on days $2-5$ (Fig. 4 e; two-way ANOVA for repeated measurements followed by Dunnett's multiple comparisons test, day 2: $P=0.018$, day 3: $P=0.038$, day 4: $P=0.012$, day 5: $P=$ 0.002). Based on a spatial probe test, compared with the CON group, the $4.9 \%$ SEV group had decreased times for crossing the platform (Fig. 4 ; Kruskal-Wallis test followed by Dunn's multiple comparisons test, $P=0.042$ ). Differences were not significant for the OFTs or MWM tests between the CON and $3.3 \% \mathrm{SEV}$ groups.

\section{WMD induced by high-concentration sevoflurane might be associated with increased activation of microglia and astrocytes}

Iba1 is specifically expressed in microglia. In the CON group, few microglia were detected. The average number of Iba1-positive cells per field of vision was significantly increased in the $4.9 \%$ SEV group at $12 \mathrm{~h}$ post anesthesia in the corpus callosum (Fig. 5a, c; one-way ANOVA followed by Dunnett's multiple comparisons test, $P=0.026$ ), cingulum (Fig. 5a, c; one-way ANOVA followed by Dunnett's multiple comparisons test, $P=0.004$ ), and external capsule (Fig. 5a, c; Kruskal-Wallis test followed by Dunn's multiple comparisons test, $P=0.002$ ). Differences among the groups in GFAP immunoreactivity (a marker of astrocytes) was similar to differences in Iba1. GFAP immunoreactivity in the $4.9 \%$ SEV group at $12 \mathrm{~h}$ post anesthesia was higher than in the CON group in the corpus callosum (Fig. 5a, c; Kruskal-Wallis test followed by Dunn's multiple comparisons test, $P<0.001$ ) and cingulum (Fig. 5a, c; Kruskal-Wallis test followed by Dunn's multiple comparisons test, $P<0.001$ ), but not the subventricular zone (Fig. 5a, c; one-way ANOVA followed by Dunnett's multiple comparisons test). Iba-1 and GFAP immunoreactivity were not significantly different between the CON and 3.3\% SEV groups (Fig. 5).

\section{Discussion}

The potential neurotoxic effects of general anesthetics have been the focus of debate and ongoing research. Due to current techniques and advanced monitoring, preterm infants might receive anesthesia for a variety of surgical procedures and imaging studies. Epidemiological studies suggest an association between surgery with anesthesia and increased risk of neurocognitive impairments in both term and preterm infants[15, 16]. In particular, preterm infants exposed to surgery and anesthesia had more white matter injury and smaller total brain volumes[17]. Accordingly, we assessed the neuroanatomical and functional consequences in a preterm-equivalent rat model exposed to different concentrations of sevoflurane by immunostaining and behavioural tests. High-concentration ( $4.9 \%$ for $2 \mathrm{~h}$ ), but not low-concentration ( $3.3 \%$ for $2 \mathrm{~h})$, sevoflurane induced a pathology closely resembling preterm infants with diffuse WMD, including impaired oligodendrocyte maturation and myelination. We also observed long-term changes in neurobehavioral performances. Furthermore, we observed widespread microglia activation and astrogliosis in white matter, which might contribute to the hampered maturation of OLs.

Age, particularly in the paediatric range, is one of the most important factors influencing requirements for inhalational anesthetics. MAC increases with decreasing age, reaching a maximum value in full-term infants at $1-6$ months of age[18,19]. MAC for inhalational anesthetics is significantly less in preterm neonates than in full-term neonates[20]. For example, MAC is 3.28\% for sevoflurane in 2-day-old rats and up to 3.74\% in 9-day-old rats (considered similar to full-term human neonates)[14]. Clinical research demonstrated that, compared with older preterm infants, effective inspired concentrations of sevoflurane to prevent movement in response to stimulation is $50 \%$ lower in lower-aged preterm infants and induction time is shorter[21]. In addition, general anesthetics are dose-dependently neurotoxic. Therefore, we used 3.3\% (approximately $1 \mathrm{MAC}$ ) and $4.9 \%$ (approximately $1.5 \mathrm{MAC}$ ) sevoflurane to determine if sevoflurane exposure was neurotoxic to the brain in preterm-equivalent rats. Our findings indicated that $4.9 \%$, but not $3.3 \%$, sevoflurane induced brain damage in pretermequivalent rats. These results were in line with findings of previous studies on anesthesia-induced neurotoxicity that found it is concentration-dependent in foetal or full-term neonatal animals [22, 23].

Neurologic and neurodegenerative diseases in the CNS are often presented from a prevalent "neuron-centric" perspective[24] that brain disorders are due to abnormal neuronal functions or survival. Similarly, pathological presentation of anesthesia-induced neurotoxicity primarily focuses on neuronal alterations in structure and function. However, the active participation of glial cells in brain physiology and pathophysiology is increasingly recognized[25]. Glial cells may constitute the majority of cells in the CNS and are necessary for correcting neuronal development and for the functions of mature neurons. OLs are a particularly specialized type of glial cell that provide fundamental support to neuronal activity by producing the myelin sheath. The consequences of deficits and dysfunction of OLs on CNS pathology are seen in a range of neurological diseases such as Alzheimer's disease, multiple sclerosis, and strokes. Previous reports described developmental loss of OLs in the white matter region of foetal or neonatal nonhuman primates following exposure to isoflurane or propofol 
$[26,27]$. Our findings indicated that high-concentration sevoflurane exposure to preterm-equivalent rat pups impeded maturation of OLs and caused deficits in myelination, contributing to poor white matter development.

Our results supported that high-concentration sevoflurane prevented oligodendrocyte differentiation/ maturation. In the CNS, oligodendrocyte progenitors undergo a complex and precisely timed program of proliferation, migration, and differentiation before forming myelin-generating cells. Glial cells of the oligodendrocyte lineage express GABA receptors and GABA is a critical regulator of oligodendrocyte development. Drugs that affect the activity of GABA receptors might alter myelination in the nervous system if they are administered during the period when OLs develop. For instance, the anti-epileptic $\mathrm{GABA}_{\mathrm{A}}$ agonist phenobarbital decreases myelin formation when administered to rat pups prenatally or postnatally[28]. Endogenous GABA signalling exerts a negative impact on the number of oligodendrocyte lineage cells and myelination in cortical organotypic slice cultures[8]. The mechanisms behind anesthetics that act on the CNS are insufficiently understood but at least sevoflurane mimics the action of the inhibitory neurotransmitter GABA. The appearance of CNPase is one of the earliest events of differentiation and early myelination. A decreased number of CNPase-expressing early myelinating OLs was observed at P14 in the high-concentration sevoflurane group. However, the exact mechanism of effects requires further investigation as reduced GABAergic input contributes to delayed oligodendrocyte maturation and dysmyelination in mice with hypoxia-induced WMD[29].

Since mature OLs are involved in the formation of myelin sheaths by wrapping around axons in the CNS, we investigated if myelination in the brain might be destroyed by sevoflurane. MBP is a component of myelin that contributes to the formation, development, and stability of myelin sheaths. High-concentration sevoflurane administration decreased MBP immunoreactivity in white matter. Myelin sheaths prevent electrical currents from moving across nerve membranes and provide vital support to axons. Converging evidence indicates that damage to OLs and the myelin sheaths are associated with axonal dysfunction in some conditions[30]. Consistently, downregulated fluorescence intensity ratios of SMI32 to MBP show that arrested oligodendrocyte maturation leads to myelin loss and axonal injury in white matter regions.

Substantial evidence from animal studies supports that exposure to anesthetic agents during foetal or early life causes behavioural deficits in older age, as white matter tracts are pivotal for mediating the essential connectivity through which behaviour and cognition is organized. White matter integrity disruptions correlate with affective (such as anxiety-related personality aspects) and/or cognitive (such as learning and memory) deficiency in a diverse range of nervous system disorders. Our results clearly demonstrated that high-concentration sevoflurane induced poor learning and memory function, measured as MWM tests in adolescent rats. In most studies, anesthesia exposure of neonatal rats at P7 did not trigger anxiety-like features later in life[31, 32]. Our findings indicate high-concentration sevoflurane exposure in P2 preterm rats induced anxiety-like consequences in OFTs. This result may be because the white matter of 2-dayold preterm-equivalent rodents is more susceptible to insults than in 7-day-old full-term equivalent animals.

Concomitantly, widespread microgliosis and astrogliosis were observed in specific white matter regions at $12 \mathrm{~h}$ post anesthesia. Several microglial-derived inflammatory cytokines released by activated microglia are identified that lead to anesthesia-mediated neurotoxicity to the brain. In response to microglia activation, astrocytes proliferate and modify the mechanisms of microglial-mediated toxicity. The activation of microglia and astrogliosis may promote neuroinflammation, which may further compromise oligodendrocyte maturation and myelination in white matter[33]. However, further studies are needed to define how neuroinflammatory mediators and anesthetics may interact to induce WMD.

There are several limitations to be noted regarding this study. Firstly, in clinical situations, different categories of anesthetic agents are used in combination, rather than a single agent. Sevoflurane with other agents such as opioids is more generally administered than single sevoflurane at high concentration. Secondly, there are such profound differences in white matter structure and brain development between the rodent and the human. For example, a rodent brain is composed of much lower levels of white matter as compared to humans. Thus, data obtained from an animal model cannot be extrapolated and applied to humans directly.

\section{Conclusions}

In conclusion, this study documented a deleterious effect on neuroanatomy and functional outcomes of postnatal high-concentration sevoflurane exposure in normal and challenged, immature white matter in a preterm-equivalent neonatal rat. These findings have potential clinical significance because use of sevoflurane is not absolutely safe for preterm neonates. The use of high concentrations of sevoflurane during this period is not recommended.

\section{Abbreviations}

minimum alveolar concentration: MAC; white matter damage: WMD; oligodendrocytes: OLs; central nervous system: CNS; g-aminobutyric acid: GABA; open field test: OFT; Morris water maze: MWM.

\section{Declarations}

\section{- Ethical Approval and Consent to participate}

Experimental animal work used newborn Sprague-Dawley rats bred at the Laboratory Animal Care Committee of China Medical University, Shenyang, China (2016PS028K) and was in accordance with the Guidelines for the Care and Use of Laboratory Animals from the National Institutes of Health (USA). Consent to participate for human subjects is not applicable in this study.

\section{- Consent for publication}

Not applicable. 
- Availability of supporting data

All data generated and/or analyzed during this study are included in this article.

\section{- Competing interests}

The authors declare that they have no competing interests.

\section{- Funding}

This work was supported by the National Nature Science Foundation of China (No. 81671311, No.81870838), the Key Research and Development Program of Liaoning Province (No.2018225004), and the Outstanding Scientific Fund of Shengjing Hospital (No. 201708).

\section{- Authors' contributions}

ZYW and PZ conceived the study and participated in the design of the study and writing the manuscript. ZYW, QSG, XYL, and HX performed molecular/cellular experiments and statistical analyses. All authors read and approved the final manuscript.

\section{- Acknowledgements}

Not applicable.

\section{References}

1.Andropoulos DB: Effect of Anesthesia on the Developing Brain: Infant and Fetus. Fetal Diagn Ther 2018, 43:1-11.

2.Kinney MV, Lawn JE, Howson CP, Belizan J: 15 Million preterm births annually: what has changed this year? Reprod Health $2012,9.28$.

3.Havidich JE, Beach M, Dierdorf SF, Onega T, Suresh G, Cravero JP: Preterm Versus Term Children: Analysis of Sedation/Anesthesia Adverse Events and Longitudinal Risk. Pediatrics 2016, 137:e20150463.

4.Filley CM, Fields RD: White matter and cognition: making the connection. J Neurophysio/ 2016, 116.2093-2104.

5.Block RI, Magnotta VA, Bayman EO, Choi JY, Thomas JJ, Kimble KK: Are Anesthesia and Surgery during Infancy Associated with Decreased White Matter Integrity and Volume during Childhood? Anesthesiology 2017, 127:788-799.

6.Back SA: White matter injury in the preterm infant: pathology and mechanisms. Acta Neuropathol 2017, 134:331-349.

7.Saab AS, Tzvetavona ID, Trevisiol A, Baltan S, Dibaj P, Kusch K, Mobius W, Goetze B, Jahn HM, Huang W, et al: Oligodendroglial NMDA Receptors Regulate Glucose Import and Axonal Energy Metabolism. Neuron 2016, 91:119-132.

8.Hamilton NB, Clarke LE, Arancibia-Carcamo IL, Kougioumtzidou E, Matthey M, Karadottir R, Whiteley L, Bergersen LH, Richardson WD, Attwell D: Endogenous GABA controls oligodendrocyte lineage cell number, myelination, and CNS internode length. Glia 2017, 65:309-321.

9.Brohan J, Goudra BG: The Role of GABA Receptor Agonists in Anesthesia and Sedation. CNS Drugs 2017, 31:845-856.

10.Back SA, Rosenberg PA: Pathophysiology of glia in perinatal white matter injury. Glia 2014, 62:1790-1815.

11.Wang LY, Tu YF, Lin YC, Huang CC: CXCL5 signaling is a shared pathway of neuroinflammation and blood-brain barrier injury contributing to white matter injury in the immature brain. J Neuroinflammation 2016, 13:6.

12.Ren Q, Zhang XF, Yang JY: Erythropoietin reduces white matter damage in two-day-old rats exposed to hypoxic/ischemia injury. Neurol Res 2016:1-7.

13.Carty ML, Wixey JA, Reinebrant HE, Gobe G, Colditz PB, Buller KM: Ibuprofen inhibits neuroinflammation and attenuates white matter damage following hypoxia-ischemia in the immature rodent brain. Brain Res 2011, 1402:9-19.

14. Orliaguet G, Vivien B, Langeron O, Bouhemad B, Coriat P, Riou B: Minimum alveolar concentration of volatile anesthetics in rats during postnatal maturation. Anesthesiology 2001, 95:734-739.

15. Hunt RW, Hickey LM, Burnett AC, Anderson PJ, Cheong JLY, Doyle LW: Early surgery and neurodevelopmental outcomes of children born extremely preterm. Arch Dis Child Fetal Neonatal Ed 2018, 103:F227-F232.

16. Houck CS, Vinson AE: Anaesthetic considerations for surgery in newborns. Arch Dis Child Fetal Neonatal Ed 2017, 102:F359-F363.

17.Filan PM, Hunt RW, Anderson PJ, Doyle LW, Inder TE: Neurologic outcomes in very preterm infants undergoing surgery. J Pediatr 2012, 160:409-414.

18.Lerman J: Inhalation agents in pediatric anaesthesia - an update. Curr Opin Anaesthesiol 2007, 20:221-226.

19.Lerman J, Sikich N, Kleinman S, Yentis S: The pharmacology of sevoflurane in infants and children. Anesthesiology 1994, 80:814-824. 
20.LeDez KM, Lerman J: The minimum alveolar concentration (MAC) of isoflurane in preterm neonates. Anesthesiology 1987, 67:301-307.

21.Yu L, Sun H, Yao L, Feng Y, Yang B: Comparison of effective inspired concentration of sevoflurane in preterm infants with different postconceptual ages. Paediatr Anaesth 2011, 21:148-152.

22.Wang Y, Yin S, Xue H, Yang Y, Zhang N, Zhao P: Mid-gestational sevoflurane exposure inhibits fetal neural stem cell proliferation and impairs postnatal learning and memory function in a dose-dependent manner. Dev Biol 2018, 435:185-197.

23.Zhou X, Li W, Chen X, Yang X, Zhou Z, Lu D, Feng X: Dose-dependent effects of sevoflurane exposure during early lifetime on apoptosis in hippocampus and neurocognitive outcomes in Sprague-Dawley rats. Int J Physiol Pathophysiol Pharmacol 2016, 8:111-119.

24.Bercury KK, Macklin WB: Dynamics and mechanisms of CNS myelination. Dev Cell 2015, 32:447-458.

25.Chung WS, Welsh CA, Barres BA, Stevens B: Do glia drive synaptic and cognitive impairment in disease? Nat Neurosci 2015, 18:1539-1545.

26.Creeley C, Dikranian K, Dissen G, Martin L, Olney J, Brambrink A: Propofol-induced apoptosis of neurones and oligodendrocytes in fetal and neonatal rhesus macaque brain. Br J Anaesth 2013, 110 Supp/ 1:i29-38.

27.Creeley CE, Dikranian KT, Dissen GA, Back SA, Olney JW, Brambrink AM: Isoflurane-induced apoptosis of neurons and oligodendrocytes in the fetal rhesus macaque brain. Anesthesiology 2014, 120:626-638.

28.Patsalos PN, Wiggins RC: Brain maturation following administration of phenobarbital, phenytoin, and sodium valproate to developing rats or to their dams: effects on synthesis of brain myelin and other subcellular membrane proteins. J Neurochem 1982, 39:915-923.

29.Zonouzi M, Scafidi J, Li P, McEllin B, Edwards J, Dupree JL, Harvey L, Sun D, Hubner CA, Cull-Candy SG, et al: GABAergic regulation of cerebellar NG2 cell development is altered in perinatal white matter injury. Nat Neurosci 2015, 18:674-682.

30.Saab AS, Nave KA: Neuroscience: A mechanism for myelin injury. Nature 2016, 529:474-475.

31.Schilling JM, Kassan A, Mandyam C, Pearn ML, Voong A, Grogman GG, Risbrough VB, Niesman IR, Patel HH, Patel PM, Head BP: Inhibition of p75 neurotrophin receptor does not rescue cognitive impairment in adulthood after isoflurane exposure in neonatal mice. Br J Anaesth 2017, 119:465-471.

32.Chung W, Park S, Hong J, Park S, Lee S, Heo J, Kim D, Ko Y: Sevoflurane exposure during the neonatal period induces long-term memory impairment but not autism-like behaviors. Paediatr Anaesth 2015, 25:1033-1045.

33.Manso Y, Holland PR, Kitamura A, Szymkowiak S, Duncombe J, Hennessy E, Searcy JL, Marangoni M, Randall AD, Brown JT, et al: Minocycline reduces microgliosis and improves subcortical white matter function in a model of cerebral vascular disease. Glia 2018, 66.34-46.

\section{Tables}

Table 1. Physiological parameters

\begin{tabular}{cccc}
\hline & $\mathrm{CON}$ & $3.3 \% \mathrm{SEV}$ & $4.9 \% \mathrm{SEV}$ \\
\hline $\mathrm{pH}$ & $7.357 \pm 0.067$ & $7.369 \pm 0.075$ & $7.361 \pm 0.083$ \\
$\mathrm{PaCO} 2(\mathrm{mmHg})$ & $40.32 \pm 1.460$ & $40.36 \pm 1.313$ & $40.12 \pm 1.681$ \\
\hline $\mathrm{PaO} 2(\mathrm{mmHg})$ & $183.2 \pm 3.685$ & $184.4 \pm 4.003$ & $183.5 \pm 4.891$ \\
\hline $\mathrm{SaO} 2(\%)$ & $96 \pm 1.732$ & $94.6 \pm 2.408$ & $94.4 \pm 1.673$ \\
\hline Glucose $(\mathrm{mg} / \mathrm{dl})$ & $129 \pm 3.391$ & $126.2 \pm 8.843$ & $125.8 \pm 4.324$ \\
\hline
\end{tabular}

$\mathrm{PaCO} 2$ = arterial carbon dioxide tension; $\mathrm{PaO} 2$ = arterial oxygen tension; $\mathrm{SaO} 2$ = arterial oxygen saturation.

Table 2. Effect of sevoflurane exposure on the growth patterns of rat brain and body. Data are presented as mean \pm SD of 5 animals at each time point in each group.

\begin{tabular}{|c|c|c|c|c|c|c|c|c|c|c|c|}
\hline & \multicolumn{4}{|c|}{ brain weight } & \multicolumn{4}{|c|}{ body weight } & \multicolumn{3}{|c|}{ brain/body weght ratio } \\
\hline & $\mathrm{P} 2$ & P7 & $\mathrm{P} 14$ & P28 & $\mathrm{P} 2$ & P7 & $\mathrm{P} 14$ & $\mathrm{P} 28$ & P2 & P7 & P14 \\
\hline $\mathrm{CON}$ & $0.494 \pm 0.024$ & $0.576 \pm 0.026$ & $1.232 \pm 0.041$ & $1.784 \pm 0.074$ & $9.46 \pm 0.472$ & $15.7 \pm 1.095$ & $36.1 \pm 1.517$ & $88.6 \pm 2.408$ & $0.052 \pm 0.005$ & $0.037 \pm 0.004$ & $0.034 \pm 0.0$ \\
\hline $\begin{array}{c}3.3 \% \\
\text { SEV }\end{array}$ & $0.506 \pm 0.035$ & $0.550 \pm 0.026$ & $1.214 \pm 0.032$ & $1.762 \pm 0.053$ & $9.76 \pm 0.378$ & $14.6 \pm 0.962$ & $34.6 \pm 2.408$ & $90.5 \pm 2.872$ & $0.052 \pm 0.004$ & $0.038 \pm 0.001$ & $0.035 \pm 0.0$ \\
\hline $\begin{array}{l}4.9 \% \\
\text { SEV } \\
\end{array}$ & $0.496 \pm 0.021$ & $0.550 \pm 0.026$ & $1.210 \pm 0.016$ & $1.734 \pm 0.065$ & $9.08 \pm 0.390$ & $14.3 \pm 0.758$ & $33.5 \pm 1.000$ & $90.6 \pm 5.505$ & $0.054 \pm 0.004$ & $0.039 \pm 0.003$ & $0.036 \pm 0.0$ \\
\hline
\end{tabular}

\section{Figures}




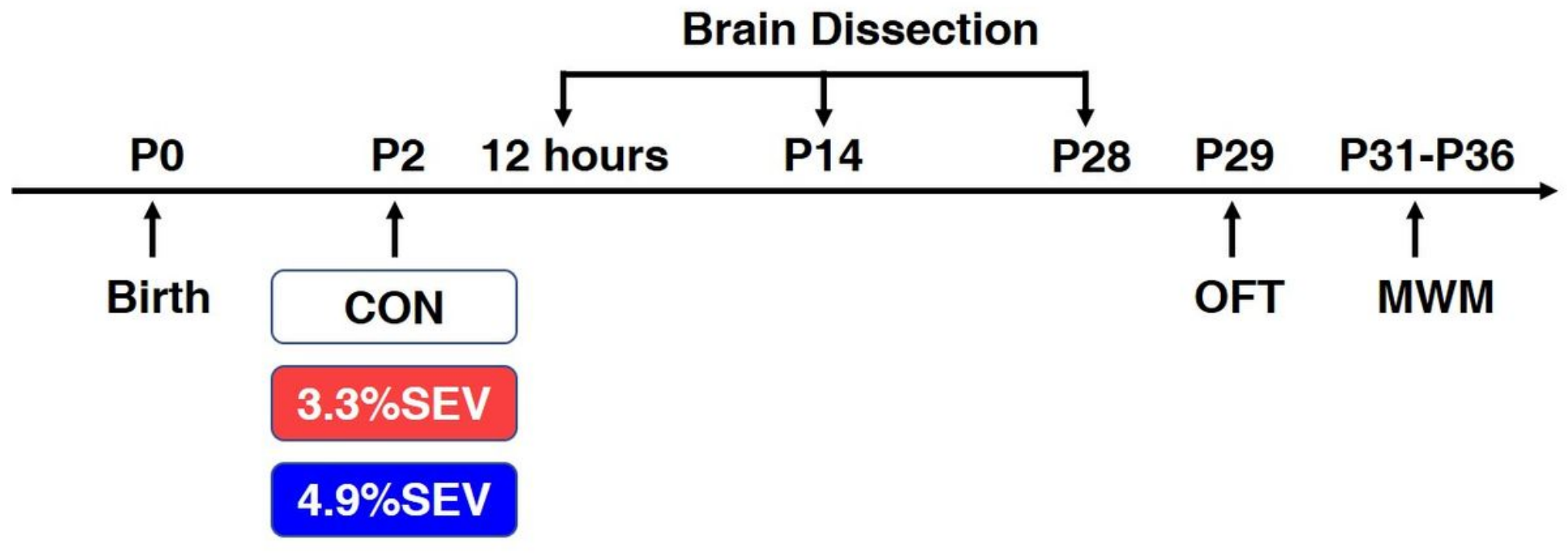

Figure 1

Diagram of experimental design. See text for details.

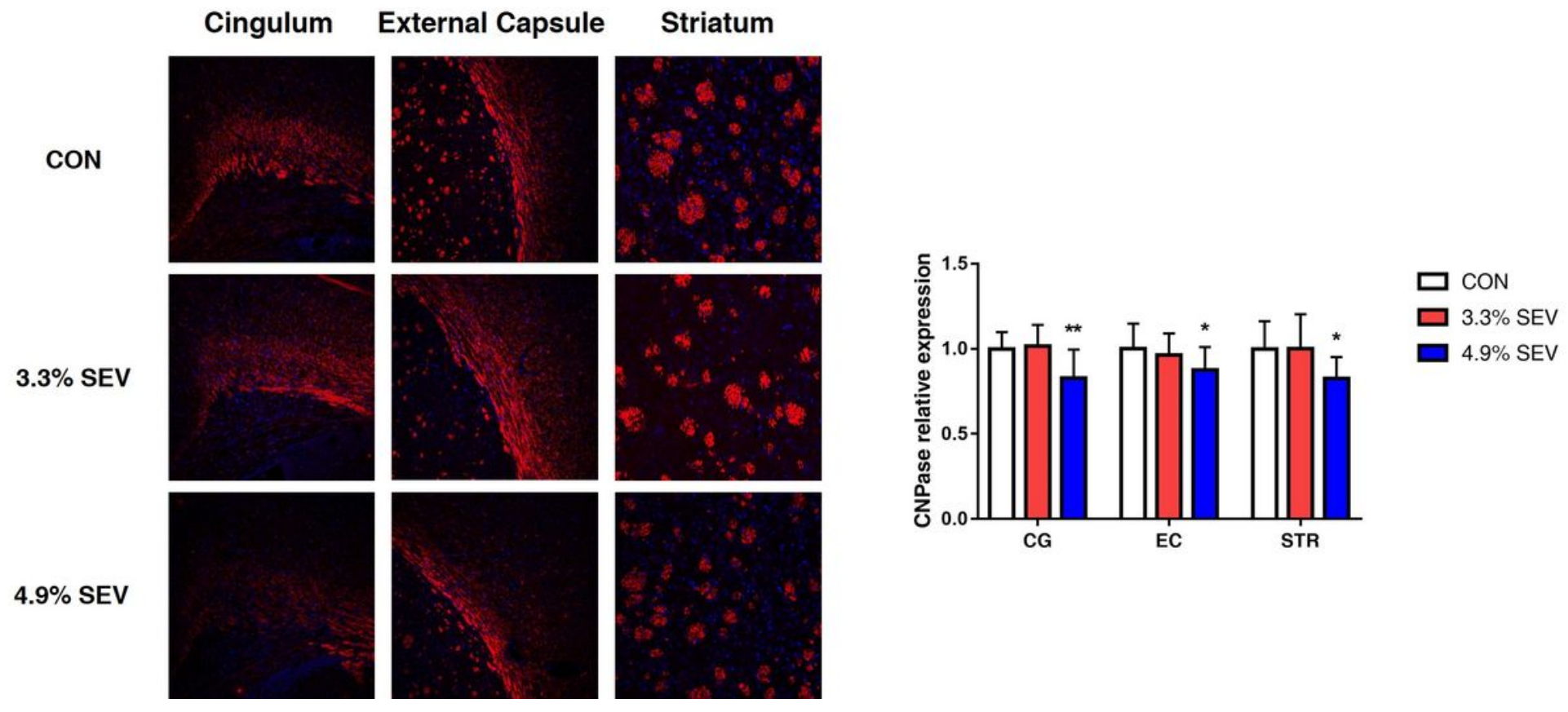

Figure 2

Effects of sevoflurane exposure on oligodendrocyte differentiation in white matter. (A) Representative images of CNPase immunostaining in the cingulum, external capsule, and striatum. Scale bar $=100 \mu \mathrm{m}$. (B) Quantification of CNPase immunostaining. Calculated as fold change over control. Data represent the means \pm SD. $n=5$ per group. ${ }^{*} P<0.05$, compared with CON group. ${ }^{* *} \mathrm{P}<0.01$, compared with CON group. One-way ANOVA followed by Dunnett's multiple comparisons test was used for data analysis. 


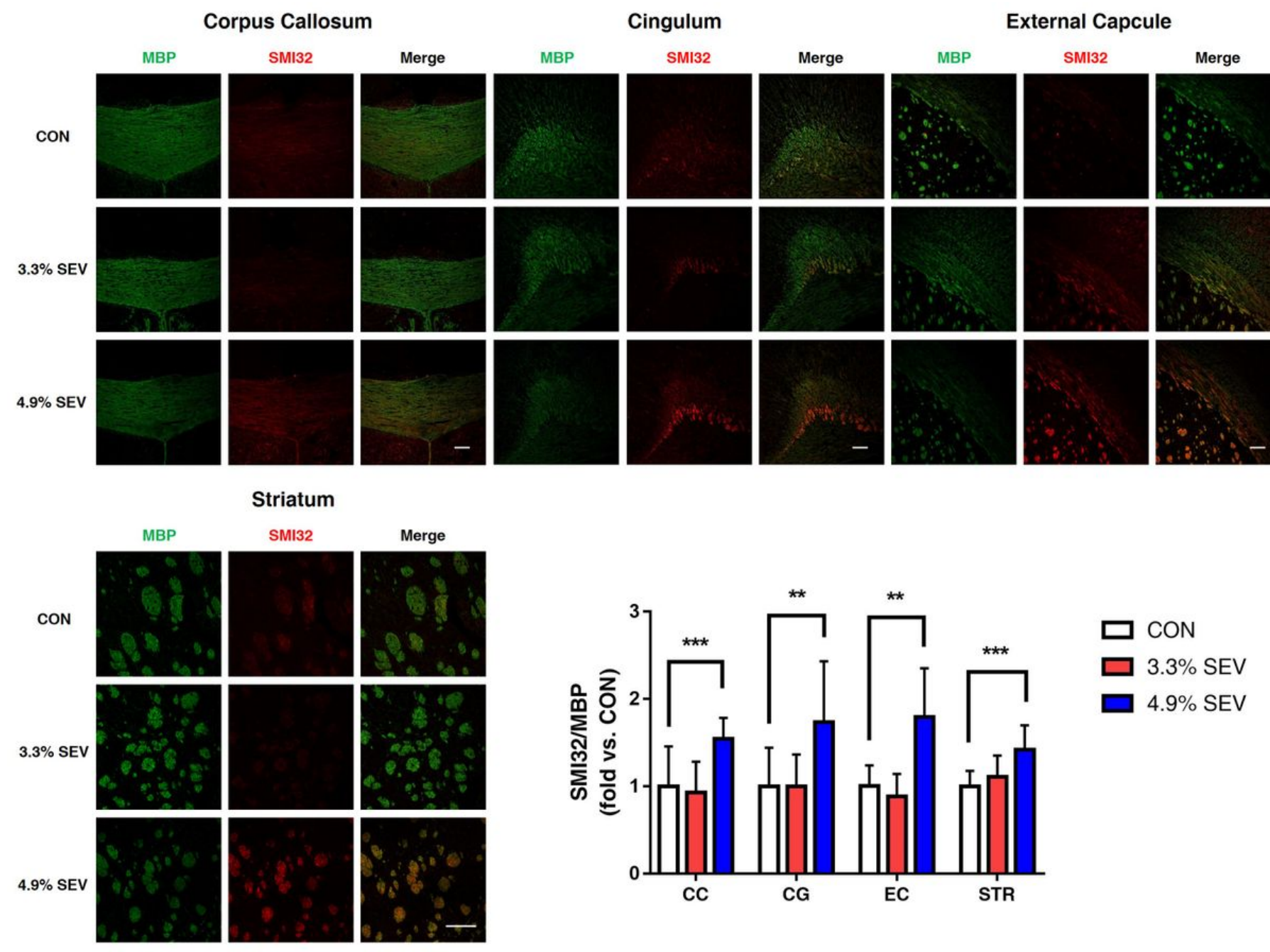

Figure 3

Effects of sevoflurane exposure on white matter integrity. (A-D) Representative images of myelin basic protein (MBP; green) and non-phosphorylated neurofilament $\mathrm{H}$ protein (SMI32; red) immunostaining in the corpus callosum (CC, A), cingulum (CG, B), external capsule (EC, C), and striatum (STR, D). Scale bar $=100 \mu \mathrm{m}$. (E) Quantification of ratio of SMI32 to MBP immunostaining. Calculated as fold change over control. Data represent the means \pm SD. $n=5$ per group. ${ }^{*} \mathrm{P}<0.01$, compared with CON group. ${ }^{* \star *} \mathrm{P}<0.001$, compared with CON group. One-way ANOVA followed by Dunnett's multiple comparisons test or Kruskal-Wallis test followed by Dunn's multiple comparisons test was used for data analysis. 


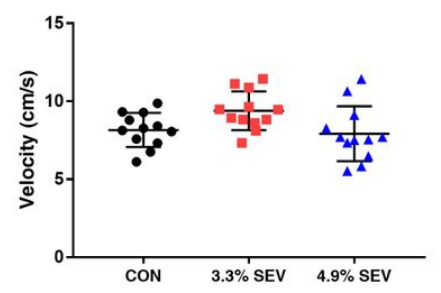

C

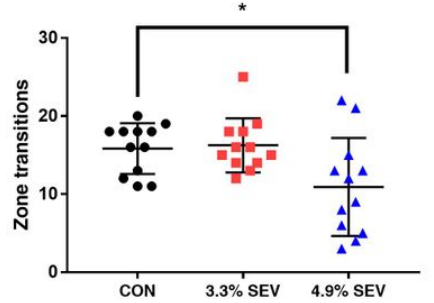

e

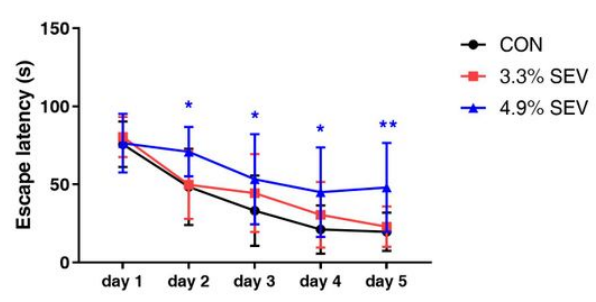

b

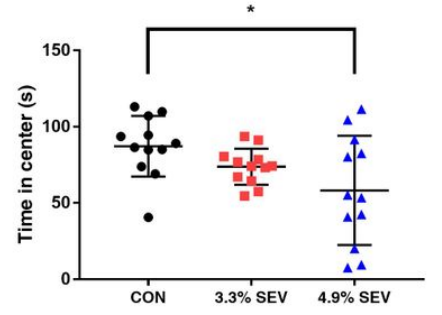

d

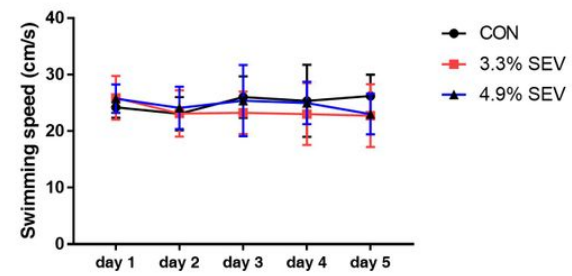

f

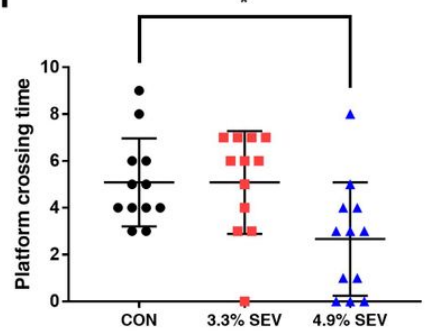

\section{Figure 4}

Evaluation of rats' behaviour in the open field test (OFT) and morris water maze (MWM) tests. (A) Velocity in 10 minutes of OFT. (B) Time in center in 10 minutes of OFT. (C) Zone transitions in 10 minutes of OFT. (D) Mean swimming speed in the MWM tests. (E) Mean escape latency of reaching the submerged platform in the training period in the MWM tests. (F) Platform crossing time in the MWM tests. $n=12$ per group. ${ }^{*}<<0.05$, compared with CON group; ${ }^{\star *} \mathrm{P}<$ 0.01, compared with CON group. Two-way ANOVA for repeated measurements followed by Dunnett's multiple comparisons test was used to determine the differences of the mean swimming speed and escape latency. One-way ANOVA followed by Dunnett's multiple comparisons test or Kruskal-Wallis test followed by Dunn's multiple comparisons test was used to determine the differences of velocity, time in center, zone transitions in the OFT and platform crossing time in the MWM tests. 


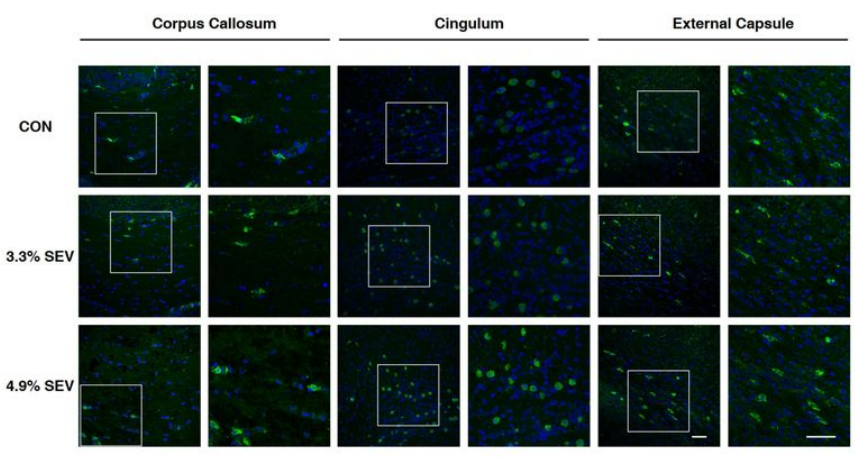

b

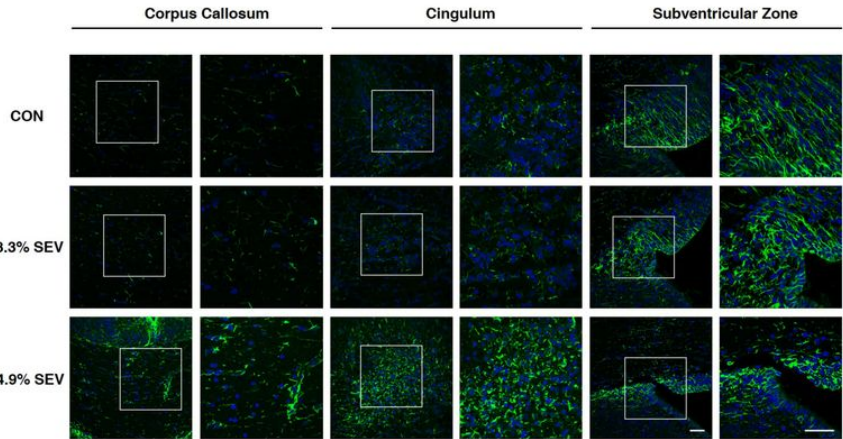

c

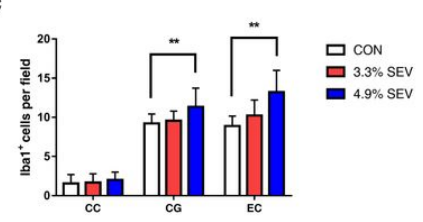

d

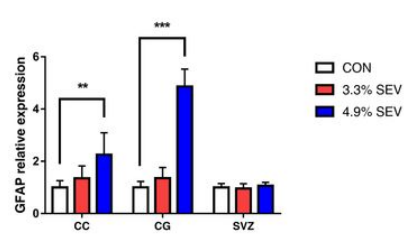

\section{Figure 5}

Effects of sevoflurane exposure on activation of microglia and astrocytes. (A) Immunofluorescence staining of Iba1, a marker for microgliosis. (B) Immunofluorescence staining of GFAP, a marker for astrogliosis. (C) Quantification of Iba1-positive cells. (D) Quantification of GFAP relative expression. $\mathrm{n}=5$ per group. Scale bar $=50 \mu \mathrm{m}$. $* * \mathrm{P}<0.01$, compared with CON group. $* * * \mathrm{P}<0.001$, compared with CON group. One-way ANOVA followed by Dunnett's multiple comparisons test or Kruskal-Wallis test followed by Dunn's multiple comparisons test was used for data analysis. 